\title{
The Fundamental Lemma: What is it and what do we know?
}

\author{
Stephen DeBacker
}

\section{Introduction}

The Fundamental Lemma (FL) was first proposed about twenty years ago by Langlands and Shelstad [29] as part of the trace-theoretic approach to the Langlands program. The FL is a rather delicate statement, which, to judge by its name, was thought to be both important and not particularly deep. The technical nature of the FL makes it rather difficult to understand and appreciate. Thus, rather than give a technical presentation full of correct definitions, we will instead attempt in this discussion to convey the flavor of the subject. As such, lies will occur ${ }^{1}$; the interested reader may consult the original papers for complete details.

Our discussion is divided into two parts. In the first part, we shall try to motivate the statement of the FL by looking at the (conjectural) Local Langlands Correspondence (LLC).

There are at least two interesting classes of distributions on a $p$-adic group: orbital integrals and characters. Although the FL is concerned with the former, most people are more comfortable with the latter. Thus, in the first part of our discussion, we will use characters and the framework of the (conjectural) LLC to introduce most of the formalism required to state the FL. The LLC, the existence of which has been proven for general linear groups by Harris-Taylor and Henniart, is a finite-to-one correspondence from the set of (equivalence classes of) irreducible smooth representations of a $p$-adic group to the set of (equivalence classes of) maps from the Weil-Deligne group into the Langlands dual group of the $p$-adic group. Verifying the existence of the LLC is the fundamental open problem in local harmonic analysis for $p$-adic

Supported by National Science Foundation Grant Number 0345121.

${ }^{1}$ I hope only intentionally. 
groups. In some sense, the FL is the analogue, for orbital integrals, of the expected behavior of the fibers of the LLC.

In the second part, we shall discuss various recent works which have approached or achieved a proof of the FL. We shall discuss the geometric approach as carried out by Laumon and Ngô, and Waldspurger's more classical, though so far not complete, approach through asymptotic expansions.

Note that there are many versions of the FL beyond the one we shall discuss. For example, there is the so-called weighted Fundamental Lemma. Except for $\mathrm{Sp}_{4}$ and general linear groups, I do not think these other versions have been established.

I am deeply indebted to Robert Kottwitz and Paul Sally, Jr. who taught me nearly all I know about harmonic analysis. I thank Loren Spice for his meticulous reading of earlier drafts of this paper; Brian Conrad for patiently explaining to me a small part of the algebraic geometry behind the geometric approach to the FL; Bao Châo Ngô for explaining his methods from a point of view that I could understand; and the referees for their corrections and suggestions. All mistakes are mine.

\section{The statement of the Fundamental Lemma}

In this part of our discussion, we attempt to present the statement of the FL in a way which will convince the reader that it is both important and natural. As a side benefit, nearly all of the words required to state the FL will appear during our discussion.

Suppose $k$ is a $p$-adic field of characteristic zero and $G$ a connected semisimple $k$-group. Further assume that $G$ is unramified $^{2}$. So, for example, $k$ could be $\mathbb{Q}_{p}$, the $p$-adic completion of $\mathbb{Q}$, and $G$ could be, among other things, a special linear group, a special unitary group splitting over an unramified extension of $k$, a symplectic group, or the exceptional group $G_{2}$. Being a $p$-adic group, $G$ has a neighborhood basis at the identity consisting of compact open subgroups.

\subsection{The (conjectural) Local Langlands Correspondence.} As usually presented, the LLC is a partition of the set of (equivalence classes of) irreducible smooth representations of $G(k)$, the group of $k$ rational points of $G$, into finite disjoint subsets, called $L$-packets, in such a way that the collection of $L$-packets is parametrized by equivalence classes of certain maps, called $L$-parameters, from the Weil-Deligne group of $k$ into the Langlands dual group of $G$ (see $\S 2.1 .3$ for a more nearly precise statement). To simplify matters, we will discuss a version of the LLC that, conjecturally, parameterizes those $L$-packets which

\footnotetext{
${ }^{2}$ That is, $G$ is $k$-quasi-split and contains a maximal $k$-torus which splits over an unramified extension of $k$.
} 
contain at least one square-integrable representation in terms of elliptic Langlands parameters. For more details about the LLC, see the wonderful expository paper [10] of Gross and Reeder.

2.1.1. Square-integrable representations. Suppose $(\pi, V)$ is a representation of $G(k)$. That is, $V$ is a complex vector space on which $G(k)$ acts through $\pi$. For $H$ a subgroup of $G(k)$, we denote by $V^{H}$ the subspace of $V$ consisting of $H$-fixed vectors. We define

$$
V^{\infty}:=\left\{v \in V: \exists \text { compact open subgroup } K \leq G(k) \text { s.t. } v \in V^{K}\right\},
$$

the space of smooth vectors in $V$. We say that $(\pi, V)$ is smooth provided that $V=V^{\infty}$; in other words, $(\pi, V)$ is smooth if $\pi(g)$ is continuous for the discrete topology on $V$, for all $g \in G(k)$.

EXAmPle 2.1.1. Let $C(G(k))$ denote the space of complex-valued functions on $G(k)$. Then $G(k)$ acts on $C(G(k))$ though the right-regular action $R$, given by $(R(g) f)(x)=f(x g)$ for $f \in C(G(k))$ and $g, x \in G(k)$. Note that $(R, C(G(k)))$ is a representation of $G(k)$, but not a smooth one (for example, characteristic functions of singletons are not fixed by any compact open subgroup of $G(k))$. The subspace $C(G(k))^{\infty}$ of smooth vectors in $C(G(k))$ is usually denoted by $C_{u}^{\infty}(G(k))$, and its elements are called uniformly locally constant functions on $G(k)$. So $C_{u}^{\infty}(G(k))$ consists of those complex-valued functions $f$ on $G(k)$ for which there exists a compact open subgroup $K=K(f)$ in $G$ so that $f(g \kappa)=f(g)$ for all $g \in G(k)$ and $\kappa \in K$.

ExAMPLE 2.1.2. We define $L^{2}(G(k))$ to be the subspace of $C_{u}^{\infty}(G(k))$ consisting of functions which are square integrable with respect to a Haar measure on $G(k)$. The group $G(k)$ acts on $L^{2}(G(k))$ through the right-regular action.

A representation $(\pi, V)$ of $G(k)$ is called irreducible provided that it is nonzero and simple as a $G(k)$-module. For example, the trivial representation $(1, \mathbb{C})$ is an irreducible representation of $G(k)$. In fact, $(1, \mathbb{C})$ is the only finite-dimensional representation of $G(k)$ that is both irreducible and smooth.

Suppose $(\pi, V)$ is a smooth representation of $G(k)$. For $v \in V$ and $\lambda \in\left(V^{*}\right)^{\infty}$, we define the matrix coefficient $m_{v, \lambda} \in C_{u}^{\infty}(G(k))$ by

$$
m_{v, \lambda}(g)=\lambda(\pi(g) v)
$$

for $g \in G(k)$. If $(\pi, V)$ is also irreducible, then every matrix coefficient of $(\pi, V)$ belongs to $L^{2}(G(k))$ if and only if one nonzero one does.

A representation $(\pi, V)$ of $G(k)$ is said to be square integrable provided that $(\pi, V)$ is irreducible and smooth, and each of its matrix coefficients belongs to $L^{2}(G(k))$. In particular, if $(\pi, V)$ is supercuspidal ${ }^{3}$, then it is square integrable. The Steinberg representation $[6, \S 8]$ (see

${ }^{3}$ A representation $(\pi, V)$ is said to be supercuspidal provided that it is smooth and irreducible, and each of its matrix coefficients has compact support. 
also [39]) is an example of a square-integrable representation which is not supercuspidal.

The set of (equivalence classes of) square-integrable representations of $G(k)$ is denoted by $\mathcal{R}^{2}(G(k))$.

2.1.2. Elliptic Langlands parameters. An elliptic Langlands parameter is a homomorphism from $W_{k} \times \mathrm{SL}_{2}(\mathbb{C})$, the Weil-Deligne group of $k$, to ${ }^{L} G$, the Langlands dual group, which satisfies many properties ${ }^{4}$.

This paper does not require an understanding of the Weil-Deligne group, or of the term "elliptic Langlands parameter"; in fact, only the boxed property (1) below is important in what follows. However, this paper does require a passing familiarity with ${ }^{L} G$ (see, for example, [3, $\S 2]$ for a fuller discussion).

Let $\hat{G}$ denote the dual group of $G$. For example, $\hat{G}$ is $\mathrm{SL}_{n}(\mathbb{C})$ for $G=\mathrm{PGL}_{n}$, is $\mathrm{SO}_{2 n+1}(\mathbb{C})$ for $G=\mathrm{Sp}_{2 n}$, and is $\mathrm{G}_{2}(\mathbb{C})$ for $G=\mathrm{G}_{2}$. The group $\hat{G}$ carries a natural action of $\Gamma$, the absolute Galois group of $k$, and the $L$-group ${ }^{L} G$ is the semi-direct product of $\hat{G}$ and $\Gamma$. We remark that the dual group of a nontrivial $k$-torus $T$ (which is never semisimple) is $\hat{T}:=\mathbf{X}^{*}(T) \otimes \mathbb{C}^{\times}$. If $T$ is a maximal $k$-torus in $G$, then $\hat{T} \leq \hat{G}$.

The only property of an elliptic Langlands parameter $\varphi$ required in the sequel is:

$$
S_{\varphi}:=C_{\hat{G}}(\varphi) \text { is finite. }
$$

2.1.3. The (conjectural) Local Langlands Correspondence. The LLC is a (conjectural) finite-to-one correspondence between $\mathcal{R}^{2}(G(k))$ and the set of $\hat{G}$-conjugacy classes of elliptic Langlands parameters. Since a bijective correspondence between countable sets is not, in and of itself, very interesting, the LLC is required to satisfy additional properties, some of which we will eventually discuss.

The elements of the partition of $\mathcal{R}^{2}(G(k))$ induced by the LLC are called (discrete series) $L$-packets. If $\varphi$ is an elliptic Langlands parameter, then we denote the associated $L$-packet by $\Pi(\varphi)$. It is conjectured that the elements of $\Pi(\varphi)$ are parameterized by the set

$$
\operatorname{Irr}^{\prime}\left(S_{\varphi}\right):=\left\{\rho \in \operatorname{Irr}\left(S_{\varphi}\right) \mid \operatorname{res}_{\hat{Z}^{\Gamma}} \rho \text { is isotrivial }\right\} .
$$

Here, $\operatorname{Irr}\left(S_{\varphi}\right)$ denotes the set (of equivalence classes) of irreducible representations of the finite group $S_{\varphi}$ of $(1) ; \operatorname{res}_{\hat{Z}^{\Gamma}} \rho$ denotes the restriction of $\rho$ to $\hat{Z}^{\Gamma}$, the group of Galois fixed points in the center of $\hat{G}$; and isotriviality of $\operatorname{res}_{\hat{Z}^{\Gamma}} \rho$ means that it is a direct sum of (a finite number of) copies of the trivial representation of $\hat{Z}^{\Gamma}$. The use of $\operatorname{Irr}^{\prime}\left(S_{\varphi}\right)$ rather 
than $\operatorname{Irr}\left(S_{\varphi}\right)$ arises from the fact that the group ${ }^{L} G$ cannot distinguish ${ }^{5}$ among the inner forms of $G$.

Suppose that $\Pi(\varphi)$ may be so parametrized. If $\rho \in \operatorname{Irr}^{\prime}\left(S_{\varphi}\right)$, then we denote by $\pi(\varphi, \rho)$ the corresponding element of $\Pi(\varphi)$. For later purposes, it is important to discuss how one may, conjecturally, fix a base point in $\Pi(\varphi)$ (that is, a distinguished element $\pi(\varphi, 1)$ of $\Pi(\varphi)$ corresponding to the trivial representation $1 \in \operatorname{Irr}^{\prime}\left(S_{\varphi}\right)$.).

2.2. Fixing a base point. We need a bit of preparation.

2.2.1. Strongly regular semisimple elements. An element ${ }^{6}$ of $G$ is called semisimple provided that it belongs to a torus in $G$. An element of $G$ is said to be strongly regular semisimple provided that $C_{G}(\gamma)$ is a torus. For example, the element

$$
\left(\begin{array}{ll}
x & 0 \\
0 & x^{-1}
\end{array}\right)
$$

in $\mathrm{SL}_{2}$ is strongly regular semisimple for $x \notin\{1,-1\}$. On the other hand, the class in $\mathrm{PGL}_{2}$ represented by the matrix

$$
\left(\begin{array}{cc}
1 & 0 \\
0 & -1
\end{array}\right)
$$

is not strongly regular semisimple, even though the connected component of its centralizer in $\mathrm{PGL}_{2}$ is a torus.

The $k$-variety of strongly regular semisimple elements in $G$ is denoted by $G^{r}$. The set $G^{r}(k)$ is dense in $G(k)$ (see, for example, [16, $\S 2.5])$.

2.2.2. Characters. Suppose $(\pi, V) \in \mathcal{R}^{2}(G(k))$. For $f$ in $C_{c}^{\infty}(G(k))$, the space of locally constant, compactly supported functions on $G(k)$, we define the operator $\pi(f) \in \operatorname{End}(V)$ by

$$
\pi(f) v:=\int_{G(k)} f(g) \pi(g) v d g
$$

for $v \in V$. Here $d g$ is a fixed Haar measure on $G(k)$.

Since $(\pi, V)$ is smooth and irreducible, it is admissible [17]; that is, for every compact open subgroup $K$ of $G(k)$, the space $V^{K}$ of $K$ fixed vectors is finite dimensional. It follows that the operator $\pi(f)$

${ }^{5}$ The Langlands dual groups for both $U(2)$ and $U(1,1)$ (which are not semisimple) may be identified with $\mathrm{GL}_{2}(\mathbb{C}) \rtimes \mathbb{Z}_{2}$, where the nontrivial element of $\mathbb{Z}_{2}$ acts on $\mathrm{GL}_{2}(\mathbb{C})$ by $x \mapsto J\left({ }^{t} x\right)^{-1} J^{-1}$, with $J$ any off-diagonal matrix in $\mathrm{GL}_{2}(\mathbb{C})$ of order two. Thus, a proper formulation of the LLC would have $L$-packets that consisted not of (equivalence classes of) representations of $G(k)$, but of (equivalence classes of) pairs $\left(G^{\prime}, \pi^{\prime}\right)$ where $G^{\prime}$ is an inner form of $G$ and $\pi^{\prime}$ is an irreducible representation of $G^{\prime}(k)$. From the Kottwitz isomorphism $[\mathbf{2 3}, \mathbf{2 2}]$, requiring that the restriction of $\rho$ to (the component group of) $\hat{Z}^{\Gamma}$ be isotrivial should, conjecturally, force $G^{\prime}=G$ (as "pure" inner forms). See [10] for a fuller exposition.

${ }^{6}$ We sometimes identify $G$ with its group of $\bar{k}$-points where $\bar{k}$ denotes a fixed algebraic closure of $k$. 
has finite rank. We can therefore define the character distribution $\Theta_{\pi}: C_{c}^{\infty}(G(k)) \rightarrow \mathbb{C}$ by

$$
\Theta_{\pi}(f):=\operatorname{tr}(\pi(f))
$$

for $f \in C_{c}^{\infty}(G(k))$. Thanks to Harish-Chandra [15], we know that there is a locally constant function, also called $\Theta_{\pi}$, on $G^{r}(k)$ such that

$$
\Theta_{\pi}(f)=\int_{G(k)} \Theta_{\pi}(g) f(g) d g
$$

for all $f \in C_{c}^{\infty}(G(k))$. The function $\Theta_{\pi}$ is independent of the choice of the Haar measure $d g$.

The function $\Theta_{\pi}$ is called the character of $\pi$. This terminology may be justified by the equality

$$
\Theta_{\pi}(\gamma)=\lim _{K \rightarrow\{1\}} \Theta_{\pi}\left(f_{\gamma, K}\right)=\lim _{K \rightarrow\{1\}} \operatorname{tr}\left(\pi\left(f_{\gamma, K}\right)\right) .
$$

Here, $\gamma \in G^{r}(k)$, the limit is over compact open subgroups $K$ which "shrink" to the identity, and $f_{\gamma, K}$ denotes the characteristic function of the set $\gamma K$ scaled by meas $_{d g}(K)^{-1}$, the inverse of the measure of $K$.

2.2.3. Nilpotent elements. Let $\mathfrak{g}$ denote the Lie algebra of $G$ and let $\mathfrak{g}(k)$ denote the $k$-points of $\mathfrak{g}$. An element $X \in \mathfrak{g}(k)$ is said to be nilpotent provided that 0 is contained in the $p$-adic closure of ${ }^{G(k)} X$, the $G(k)$-orbit of $X$. We let $\mathcal{N}$ denote the set of nilpotent elements in $\mathfrak{g}(k)$. The group $G(k)$ acts on $\mathcal{N}$, and we let $\mathcal{O}(0)$ denote the set of nilpotent orbits. Since $k$ has characteristic zero, the cardinality of $\mathcal{O}(0)$ is finite.

EXAmPLE 2.2.1. Suppose $G=\mathrm{SL}_{2}$. As $\theta$ ranges over representatives for $k /\left(k^{\times}\right)^{2}$, the matrices

$$
\left(\begin{array}{ll}
0 & \theta \\
0 & 0
\end{array}\right)
$$

range over a complete set of representatives for the orbits in $\mathcal{O}(0)$. If $p \neq 2$, then the cardinality of $\mathcal{O}(0)$ is 5 .

Recall that $G$ is $k$-quasi-split. The regular nilpotent orbits are the elements of maximal dimension in $\mathcal{O}(0)$. Naively, one might think that there was only one regular nilpotent orbit. However, already in the example of $\mathrm{SL}_{2}$ above, we see that the number of regular nilpotent orbits is greater than one.

Fix, once and for all, a regular nilpotent orbit $\mathcal{O}_{m}$.

2.2.4. Base point. According to the Harish-Chandra-Howe local character expansion (see, for example, $[\mathbf{1 5}]$ ), the character $\Theta_{\pi}$ of an irreducible smooth representation $(\pi, V)$ of $G(k)$ has, in some neighborhood of zero, an asymptotic expansion of the form

$$
\Theta_{\pi} \circ \exp =\sum_{\mathcal{O} \in \mathcal{O}(0)} c_{\mathcal{O}}(\pi) \cdot \hat{\mu}_{\mathcal{O}}
$$

where the $c_{\mathcal{O}}(\pi)$ 's are complex numbers and the $\hat{\mu}_{\mathcal{O}}$ 's are class functions on $\mathfrak{g}(k)$ which are independent of $\pi$. Thanks to a result of Rodier [38] 
(see also $[\mathbf{3 3}]$ ), a conjecture of Shahidi $[\mathbf{4 1}]$ may be recast to assert that the bijective correspondence $\rho \mapsto \pi(\varphi, \rho)$ can be chosen so that $c_{\mathcal{O}_{m}}(\pi(\varphi, 1)) \neq 0$, and $c_{\mathcal{O}_{m}}(\pi(\varphi, \rho))=0$ for $\rho \neq 1$ We assume that this conjecture of Shahidi is true, and choose the base point in $\Pi(\varphi)$ accordingly.

2.3. Endoscopy. Having fixed the base point, it is natural to ask: How canonical is the correspondence $\rho \mapsto \pi(\varphi, \rho)$ ? This question also has a conjectural answer, which we now discuss. Recall that we have placed various assumptions on $G$ and $\varphi$.

2.3.1. Endoscopic groups. For a precise discussion of endoscopic groups (and their associated technicalities) see, for example, [26, Chapter 2] or $[\mathbf{4 5}, \S 2]$.

Suppose $s \in S_{\varphi} \leq \hat{G}$. We want to associate to the pair $(\varphi, s)$ an (unramified) endoscopic group. Let $\hat{H}$ denote the connected component of the centralizer of $s$ in $\hat{G}$. This will be a connected reductive $\mathbb{C}$ group. The goal is to produce an unramified $k$-group $H$ and an elliptic Langlands parameter $\varphi_{H}$ from the Weil-Deligne group into ${ }^{L} H$ such that the connected component of ${ }^{L} H$ is $\hat{H}$, and there is a map $\eta:{ }^{L} H \rightarrow$ ${ }^{L} G$ extending the injection of $\hat{H}$ into $\hat{G}$ so that $\eta \circ \varphi_{H}=\varphi$.

This is a bit technical (for example, $H$ need not be semisimple, or even $k$-split), and the technicalities are irrelevant for the present discussion. The group $H$ is an example of an endoscopic group of $G$. If $G$ is $\mathrm{PGL}_{2}$, then $H$ is either $\mathrm{GL}_{1}$ or $\mathrm{PGL}_{2}$. If $G$ is $\mathrm{SL}_{2}$, then $H$ is $\mathrm{SL}_{2}, \mathrm{GL}_{1}$, or $U_{E}^{1}$, where $U_{E}^{1}$ is a one-dimensional non-split torus which is split by a quadratic unramified extension $E$ of $k$ (in particular, $U_{E}^{1}(k)$ is compact). If $G$ is $\mathrm{G}_{2}$, then $H$ is $\mathrm{SO}_{4}, \mathrm{G}_{2}, \mathrm{PGL}_{3}$, or $\mathrm{GL}_{1} \times \mathrm{GL}_{1}$.

We now want to "compare" the $L$-packets $\Pi\left(\varphi_{H}\right)$ in $\mathcal{R}^{2}(H(k))$ and $\Pi(\varphi)$ in $\mathcal{R}^{2}(G(k))$. In order to do this, we need some way to compare $H(k)$-conjugacy classes in $H(k)$ and $G(k)$-conjugacy classes in $G(k)$.

2.3.2. Stable conjugacy: Definition. We begin with a warning. Two elements $\gamma, \gamma^{\prime} \in G^{r}(k)$ can be $G$-conjugate without being $G(k)$-conjugate. This is perhaps easiest to see at the level of the Lie algebra.

ExAmple 2.3.1. Suppose $R \subset k$ is the ring of integers and $\varpi$ is a uniformizer in $R$; that is, $\varpi R$ is the prime ideal in $R$. We assume $p \neq 2$ and fix $\varepsilon \in R^{\times} \backslash\left(R^{\times}\right)^{2}$. The elements

$$
\left(\begin{array}{ll}
0 & 1 \\
\varepsilon & 0
\end{array}\right) \text { and }\left(\begin{array}{cc}
0 & \varpi \\
\varpi^{-1} \varepsilon & 0
\end{array}\right)
$$

in $\mathfrak{s l}_{2}(k)$ are $\mathrm{SL}_{2}$-conjugate, but not $\mathrm{SL}_{2}(k)$-conjugate.

For $\gamma \in G^{r}(k)$, we denote by ${ }^{G} \gamma$ the full geometric orbit of $\gamma$ in $G$. We call $\left({ }^{G} \gamma\right)(k)={ }^{G} \gamma \cap G(k)$ the stable conjugacy class of $\gamma$. See $[\mathbf{2 1}]$ for a fuller discussion of the notion of stable conjugacy. 
2.3.3. Stable conjugacy: $H$ versus $G$. See $[\mathbf{2 9}, \S 1.3]$ for a fuller discussion of the material in this section.

Fix a maximal $k$-torus $T_{H}$ in $H$. Let $\hat{T}_{H}$ denote the corresponding dual torus in $\hat{H}$.

If $H^{s s}$ denotes the set of semisimple elements in $H$, then there is a natural isomorphism of $k$-varieties

$$
H^{s s} / H:=H^{s s} / H \text {-conjugacy } \cong T_{H} / W_{H},
$$

where $W_{H}$ is the Weyl group $N_{H}\left(T_{H}\right) / T_{H}$, and $T_{H} / W_{H}$ denotes the quotient of $T_{H}$ by the action of $W_{H}$. One may think of $H^{s s} / H$ as the set of semisimple conjugacy classes in $H$.

Since $s \in \hat{T}_{H} \leq \hat{H}$, we can, by, for example, [36], find a maximal $k$-torus $T_{G}$ in $G$ so that $T_{H}$ is $k$-isomorphic to $T_{G}, \hat{T}_{G}=\hat{T}_{H}$, etc. We then have a (canonical) $\Gamma$-map

$$
\Delta_{H}^{G}: H^{s s} / H \cong T_{H} / W_{H} \rightarrow T_{G} / W_{G} \cong G^{s s} / G .
$$

Naively, one would think that, at the level of $k$-points, the map $\Delta_{H}^{G}$ would induce a finite-to-one (though not generally surjective) map from the set of stable conjugacy classes in $H(k)$ into the set of stable conjugacy classes in $G(k)$. (Recall that a stable conjugacy class is the set of rational points of the orbit of a strongly regular semisimple element.) This is almost true, except that the image of a stable conjugacy class in $H(k)$ need not be a subset of $G^{r}(k)$.

To correct this difficulty, we introduce another definition. An element $\gamma_{H} \in H^{r}(k)$ is said to be strongly G-regular semisimple if the image of $\left({ }^{H} \gamma\right)(k)$ is a subset of $G^{r}(k)$. We let $H^{G}(k)$ denote the (dense) subset of strongly $G$-regular semisimple elements in $H^{r}(k)$. For $\gamma_{H} \in H^{G}(k)$ and $\gamma \in G^{r}(k)$, we write $\gamma_{H} \mapsto \gamma$ provided that the image of $\left({ }^{H} \gamma_{H}\right)(k)$ is $\left({ }^{G} \gamma\right)(k)$.

For $s$ as above, we define

$$
\Theta_{\varphi, s}=\Theta_{\varphi, s}^{G}:=\sum_{\rho \in \operatorname{Irr}^{\prime}\left(S_{\varphi}\right)} \operatorname{tr}(\rho(s)) \cdot \Theta_{\pi(\varphi, \rho)} .
$$

It is conjectured that the function $\Theta_{\varphi, 1}$ is stable, that is, that we have $\Theta_{\varphi, 1}(\gamma)=\Theta_{\varphi, 1}\left(\gamma^{\prime}\right)$ whenever $\gamma, \gamma^{\prime} \in G^{r}(k)$ are stably conjugate. We assume this.

We can now state the conjectural answer to the question: How canonical is the correspondence $\rho \mapsto \pi(\varphi, \rho)$ ? It is conjectured (see, for example, [28]) that, for each $s \in S_{\varphi}$ and each $\gamma \in G^{r}(k)$, we have

$$
\begin{aligned}
\left|D_{G}(\gamma)\right|^{1 / 2} \sum_{\gamma^{\prime}} \Theta_{\varphi, s}^{G}\left(\gamma^{\prime}\right) & \\
& =\sum_{\gamma_{H} \mapsto \gamma}\left|D_{H}\left(\gamma_{H}\right)\right|^{1 / 2} \cdot \Delta^{\mathrm{spec}}\left(\gamma, \gamma_{H}\right) \cdot \Theta_{\varphi_{H}, 1}^{H}\left(\gamma_{H}\right) .
\end{aligned}
$$


The elements $\gamma^{\prime}$ are representatives for the rational conjugacy classes in the $G$-stable conjugacy class of $\gamma$. The elements $\gamma_{H}$ are representatives for the $H$-stable classes in $H^{G}(k)$ which map to the $G$-stable class of $\gamma$. Since characters are linearly independent [18, Lemma 7.1], this conjectural equality would uniquely determine the correspondence $\rho \mapsto \pi(\varphi, \rho) . \quad D_{G}$ and $D_{H}$ are the discriminant functions on $G$ and $H$. The function $\Delta^{\operatorname{spec}}(\cdot, \cdot)$ on $G^{r}(k) \times H^{G}(k)$ is called a (spectral) transfer factor; we will have more to say about such functions later. For now, we note that $\Delta^{\text {spec }}\left(\gamma, \gamma_{H}\right)$ is nonzero exactly when $\gamma_{H} \mapsto \gamma$; $\Delta^{\text {spec }}\left(\gamma, \gamma_{H}\right)$ depends only on the $H$-stable conjugacy class of $\gamma_{H}$ and the $G(k)$-conjugacy class of $\gamma$; and, when both quantities are nonzero, the quotient of $\Delta^{\operatorname{spec}}\left(\gamma, \gamma_{H}\right)$ by $\Delta^{\operatorname{spec}}\left(\gamma, \gamma_{H}^{\prime}\right)$ is a root of unity.

2.4. Orbital integrals and the Fundamental Lemma. As discussed in the introduction, there is another interesting class of $G(k)$ invariant distributions on $G(k)$ : the set of orbital integrals. The FL is an analogue of (2) with the characters replaced by orbital integrals. Since orbital integrals are not represented by locally integrable functions, the analogue of (2) needs to take a different form: instead of evaluating at well chosen elements $\gamma_{H}$ and $\gamma^{\prime}$, we will evaluate at well chosen functions.

2.4.1. Strongly regular orbital integrals. Suppose $\gamma \in G^{r}(k)$. Let ${ }^{G(k)} \gamma:=\left\{g \gamma g^{-1} \mid g \in G(k)\right\}$ denote the $G(k)$-orbit of $\gamma$, and $C_{c}^{\infty}\left({ }^{G(k)} \gamma\right)$ denote the set of locally constant, compactly supported functions on ${ }^{G(k)} \gamma$. Since $\gamma$ is semisimple, we have that ${ }^{G(k)} \gamma$ is closed in $G(k)$. Thus, the map $f \mapsto \operatorname{res}_{G(k)} \gamma$ induces a map $C_{c}^{\infty}(G(k)) \rightarrow C_{c}^{\infty}(G(k) \gamma)$. It therefore makes sense to define the (normalized) orbital integral of $f \in C_{c}^{\infty}(G(k))$ by

$$
\mu_{\gamma}^{G}(f):=\left|D_{G}(\gamma)\right|^{1 / 2} \int_{G(k) \gamma} f(x) d \mu_{\gamma}(x),
$$

where $d \mu_{\gamma}^{G}$ denotes the unique (up to scaling) $G(k)$-invariant measure on ${ }^{G(k)} \gamma \cong G(k) / C_{G(k)}(\gamma)=G(k) /\left(C_{G}(\gamma)\right)(k)$ and $D_{G}$ is as above.

2.4.2. A statement of the FL. We can now state the FL: For an endoscopic group $H$ of $G$ and a strongly $G$-regular semisimple element $\gamma_{H} \in H(k)$, we have

$$
\sum_{\gamma^{\prime} \sim \gamma_{H}} \mu_{\gamma^{\prime}}^{H}\left(\left[K_{H}\right]\right)=\sum_{\gamma \sim \gamma_{H}} \Delta\left(\gamma, \gamma_{H}\right) \cdot \mu_{\gamma}^{G}\left(\left[K_{G}\right]\right) .
$$

Here, the left-hand sum runs over representatives for the $H(k)$-conjugacy classes in the $H$-stable class of $\gamma_{H}$. On the right-hand side, $\gamma$ runs over representatives for the $G(k)$-conjugacy classes in the image under $\Delta_{H}^{G}$ of the $H$-stable class of $\gamma_{H}$. Since both $G$ and $H$ are unramified, we may assume that they are defined over $R$, the ring of integers of $k$. We may then take $K_{H}$ (resp., $K_{G}$ ) to be the compact open subgroup 
$H(R)$ (resp., $G(R))$. We let $\left[K_{H}\right]$ (resp., $\left[K_{G}\right]$ ) denote the characteristic function of $K_{H}$ (resp., $K_{G}$ ).

Finally, a definition of the transfer factor

$$
\Delta(\cdot, \cdot): G^{r}(k) \times H^{G}(k) \rightarrow \mathbb{C}
$$

may be found in [29]. For $\left(\gamma, \gamma_{H}\right) \in G^{r}(k) \times H^{G}(k)$, it has the properties:

(1) $\Delta\left(\gamma, \gamma_{H}\right)$ is zero unless $\gamma_{H} \mapsto \gamma$.

(2) If $\gamma_{H}$ and $\gamma_{H}^{\prime}$ are stably conjugate, then $\Delta\left(\gamma, \gamma_{H}\right)=\Delta\left(\gamma, \gamma_{H}^{\prime}\right)$.

(3) If $\gamma$ and $\gamma^{\prime}$ are $G(k)$-conjugate, then $\Delta\left(\gamma, \gamma_{H}\right)=\Delta\left(\gamma^{\prime}, \gamma_{H}\right)$.

One of the ingredients in the definition of $\Delta(\cdot, \cdot)$ is Tate-Nakayama duality. Suppose $\gamma \in G^{r}(k)$. Let $S$ denote the torus $C_{G}(\gamma)$. There are elements $\gamma_{i} \in G(k)$ such that

$$
{ }^{G} \gamma \cap G(k)=\coprod_{i}{ }^{G(k)} \gamma_{i},
$$

where $i$ runs over $\operatorname{ker}\left(\mathrm{H}^{1}(k, S) \rightarrow \mathrm{H}^{1}(k, G)\right)$. From Tate-Nakayama duality, we have that

$$
\mathrm{H}^{1}(k, S) \cong \operatorname{Irr}\left(\pi_{0}\left(\hat{S}^{\Gamma}\right)\right) .
$$

Here $\operatorname{Irr}\left(\pi_{0}\left(\hat{S}^{\Gamma}\right)\right)$ denotes the set of irreducible representations of the component group of the $\Gamma$-fixed points in $\hat{S}$. If $\gamma_{H} \mapsto \gamma$, then we can assume $s \in \hat{S}^{\Gamma}$. Thus $s$ defines a character $\kappa_{s}$ on $\mathrm{H}^{1}(k, S)$, and $\Delta\left(\gamma_{i}, \gamma_{H}^{\prime}\right)$ is essentially $\kappa_{s}(i)$. Under these conditions, the right-hand side of $(3)$ is often written as

$$
\text { const } \cdot \mu_{\gamma}^{\kappa_{s}}\left(\left[K_{G}\right]\right)
$$

where const is a constant and $\mu_{\gamma}^{\kappa_{s}}$ is the "kappa" orbital integral $\sum_{i} \kappa_{s}\left(\gamma_{i}\right) \cdot \mu_{\gamma_{i}}^{G}$.

Warning: we have not worried about a base point in the orbital integral setting. We will return to this point later.

\section{What we know}

We now turn our attention to the current state of knowledge about the validity of the FL. Via global arguments, the exponential map, and results of Langlands and Shelstad [30], Waldspurger [44, 45] (see also [12]) has shown that, if (for all unramified groups and almost all $p$ ) the FL for the Lie algebra (see below for statement) holds, then the FL is true (see [24] for a terrific discussion of this and of transfer factors on the Lie algebra). Consequently, nearly all recent work in the area has concentrated on verifying the FL for the Lie algebra. The most general (published) result obtained so far is due to Waldspurger [45]. It says that the FL holds for any reductive $k$-group $G^{\prime}$ which splits over an unramified extension of $k$ when the endoscopic group $H$ under consideration is the $k$-quasi-split inner form of $G^{\prime}$. Ngô has recently announced a general proof of the FL for the Lie algebra [35]. 
3.1. Reduction to the Lie algebra. In this section, we outline how to reduce the proof of the FL to that of the FL for the Lie algebra, which is stated in Section 3.1.3.

3.1.1. Reduction to topologically unipotent elements. Let $q$ denote the order of $\mathfrak{f}$, the residue field of $k$. An element ${ }^{7} g \in G(k)$ for which $g^{q^{n}} \rightarrow 1$ is called topologically unipotent. We denote by $G(k)_{0^{+}}$the set of topologically unipotent elements in $G(k)$.

Suppose $\gamma \in G^{r}(k)$. If $\mu_{\gamma}^{G}\left(\left[K_{G}\right]\right) \neq 0$, then ${ }^{G(k)} \gamma \cap K_{G}$ is nonempty. So, when considering the FL, for purposes of computing orbital integrals we may, without loss of generality, assume $\gamma \in K_{G}$.

Choose $m$ so that every semisimple element of $G(\mathfrak{f})$, the reductive quotient of $K_{G}$, has order dividing $q^{m}-1$. Since $\gamma$ lies in $K_{G}$, it has a topological Jordan decomposition; that is, we may write $\gamma=\gamma_{s} \gamma_{u}=$ $\gamma_{u} \gamma_{s}$, where

$$
\gamma_{s}=\lim _{n \rightarrow \infty} \gamma^{q^{m n}}
$$

and

$$
\gamma_{u}=\gamma \gamma_{s}^{-1}
$$

are the topologically semisimple and topologically unipotent (not semisimple and unipotent) parts of $\gamma$ (see, for example, [43]). The element $\gamma_{u}$ belongs to $G(k)_{0^{+}}$. It is also true that $\gamma_{u}$ is a strongly regular semisimple element of $C_{G}\left(\gamma_{s}\right)^{\circ}(k)$, the group of $k$-rational points of the identity component of the centralizer of $\gamma_{s}$ in $G$.

$$
\text { If } g \in G(k) \text { and }{ }^{g} \gamma \in K_{G} \text {, then } g \in K_{G} \cdot C_{G(k)}\left(\gamma_{s}\right) \text { (see, }
$$
for example, $[\mathbf{1 9}, \mathbf{2 3}])$; so it is believable that $\mu_{\gamma}^{G}\left(\left[K_{G}\right]\right)$ is related to

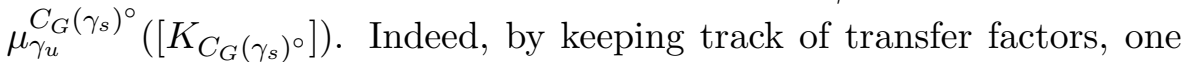
can reduce the problem of checking the FL to that of checking it on the set of topologically unipotent elements $[\mathbf{1 1}, \mathbf{3 0}]$ in $G(k)$ and $H(k)$.

3.1.2. Exponentiation. From our discussion of topologically unipotent elements in Section 3.1.1, we see that it is enough to verify the FL for strongly $G$-regular semisimple $\gamma_{H} \in H(k)_{0^{+}}$.

We shall use the exponential map to transfer the problem to the Lie algebra. Let $\mathfrak{g}(k)_{0^{+}}$denote the set of topologically nilpotent elements ${ }^{8}$ in $\mathfrak{g}(k)$. For example, if $\mathfrak{g}=\mathfrak{s l}_{n}$, then $X \in \mathfrak{g}(k)_{0^{+}}$if and only if each of the eigenvalues of $X$ has positive valuation.

Under suitable conditions on $q$, the exponential map defines a bijective, $G(k)$-equivariant map from $\mathfrak{g}(k)_{0^{+}}$to $G(k)_{0^{+}}$(see, for example, [7, Appendix B]).

${ }^{7}$ We also require $g$ to lie in some parahoric subgroup of $G(k)$.

${ }^{8}$ In the notation of [34],

$$
\mathfrak{g}(k)_{0^{+}}=\bigcup_{x \in \mathcal{B}(G)} \mathfrak{g}(k)_{x, 0^{+}} .
$$


3.1.3. Statement of the FL for the Lie algebra. An element of $\mathfrak{g}(k)$ is called regular semisimple provided that its centralizer in $G$ is a torus ${ }^{9}$. We let $\mathfrak{g}^{r}(k)$ denote the set of regular semisimple elements in $\mathfrak{g}(k)$.

The discussion in Section 2.3.3 showing how to relate stable orbits in $H$ and $G$ carries over to the Lie algebras $\mathfrak{h}$ and $\mathfrak{g}$. In particular, we denote by $\mathfrak{h}^{G}(k)$ the set of $G$-regular elements in $\mathfrak{h}^{r}(k)$ and, for $X_{H} \in \mathfrak{h}^{G}(k)$ and $X \in \mathfrak{g}^{r}(k)$, write $X_{H} \mapsto X$ whenever the stable orbit of $X$ is the image of the stable orbit of $X_{H}$.

Just as we defined orbital integrals on $G(k)$, we may, for $X \in \mathfrak{g}^{r}(k)$, define the normalized orbital integral $\mu_{X}^{G}$, a distribution on $\mathfrak{g}(k)$. The FL for the Lie algebra states that

$$
\sum_{X_{H}^{\prime} \sim X_{H}} \mu_{X_{H}^{\prime}}^{H}([\mathfrak{h}(R)])=\sum_{X \sim X_{H}} \Delta\left(X, X_{H}\right) \cdot \mu_{X}^{G}([\mathfrak{g}(R)]) .
$$

Here, $X_{H}^{\prime}$ ranges over a set of representatives for $H(k)$-conjugacy classes in the stable orbit of $X_{H}$, and $X$ ranges over a set of representatives for the $G(k)$-conjugacy classes in the image of the stable orbit of $X_{H}$. The lattice $\mathfrak{h}(R)$ in $\mathfrak{h}(k)$ is the Lie algebra analogue of $K_{H}$ (and similarly for $\mathfrak{g}(R))$.

From $[\mathbf{1 2}, \mathbf{4 5}]$, the FL holds for fields of arbitrary residual characteristic if and only if it holds when the residual characteristic is sufficiently large (here, we think of $G$ as being defined over a number field of which $k$ is a completion). Thus, from the discussion in Sections 3.1.1 and 3.1.2 about topologically unipotent and nilpotent elements and the exponential map, it is not hard to imagine that the validity of the FL is equivalent to the validity of the FL on the Lie algebra. In fact, this is true.

3.1.4. Fixing a base point. In our discussion of the LLC, we (conjecturally) chose the base point $\pi(\varphi, 1)$ in $\Pi(\varphi)$ by fixing a regular nilpotent orbit $\mathcal{O}_{m}$. Before discussing the analogous choice on the Lie algebra, we must consider the question: What is the Lie algebra analogue of an $L$-packet?

If $X \in \mathfrak{g}^{r}(k)$, then we can associate two distributions to $X$ : the orbital integral $\mu_{X}^{G}$ and its Fourier transform $\hat{\mu}_{X}^{G}$. The Fourier transform ${ }^{10}$ of $\mu_{X}^{G}$ is defined by $\hat{\mu}_{X}^{G}(f):=\mu_{X}^{G}(\hat{f})$ for $f \in C_{c}^{\infty}(\mathfrak{g}(k))$, where $\hat{f}$ denotes the Fourier transform of $f$. According to Harish-Chandra [14] (see $[\mathbf{2 4}]$ for an excellent summary), for harmonic analysis on $\mathfrak{g}(k)$, the distributions $\hat{\mu}_{X}^{G}$ should be thought of as analogues of characters. The stable orbit of $X$ breaks up into a finite number of $G(k)$-conjugacy classes. Suppose $\left\{X_{1}=X, X_{2}, \ldots, X_{\ell}\right\}$ is a set of representatives for

${ }^{9}$ In characteristic zero, the centralizer of a semisimple element of the Lie algebra is always connected, so we drop the word "strongly" from our discussion.

${ }^{10}$ To define the Fourier transform in the present setting, one must fix a nontrivial additive character on $k$ and a nondegenerate, bilinear, $G(k)$-invariant form on $\mathfrak{g}(k)$. 
these classes. To each $X_{i}$, we can associate the distribution $\hat{\mu}_{X_{i}}^{G}$. Then the set $\left\{\hat{\mu}_{X_{i}}^{G} \mid 1 \leq i \leq \ell\right\}$ is the analogue of an $L$-packet.

We can now consider how to fix a base point. Recall that $\mathcal{O}_{m}$ is our fixed regular nilpotent orbit in $\mathfrak{g}(k)$. Thanks to Shelstad [42], the Lie algebra analogue of the statement that $c_{\mathcal{O}_{m}}(\pi)$ is nonzero for exactly one element $\pi$ of a tempered $L$-packet is known to be true for the " $L$-packet" $\left\{\hat{\mu}_{X_{i}}^{G} \mid 1 \leq i \leq \ell\right\}$.

This result of Shelstad translates into a very elegant solution to the problem of fixing a base point for the orbital integrals. Choose $X_{m} \in \mathcal{O}_{m}$. From the Jacobson-Morozov theorem (see, for example, [5, $\S 5.3])$, there exists a $k$-injection $\delta: \mathfrak{s l}_{2} \rightarrow \mathfrak{g}$ so that

$$
\left(\begin{array}{ll}
0 & 1 \\
0 & 0
\end{array}\right) \mapsto X_{m} .
$$

Set

$$
Y_{m}=\delta\left(\left(\begin{array}{ll}
0 & 0 \\
1 & 0
\end{array}\right)\right)
$$

and consider the Kostant section $V=X_{m}+C_{\mathfrak{g}(k)}\left(Y_{m}\right)$. For example, for $\mathfrak{g}=\mathfrak{s l}_{3}$,

$$
X_{m}=\left(\begin{array}{lll}
0 & 1 & 0 \\
0 & 0 & 1 \\
0 & 0 & 0
\end{array}\right),
$$

and the appropriate choice of $Y_{m}$, we have that $V$ is the set of matrices of the form

$$
\left(\begin{array}{lll}
0 & 1 & 0 \\
a & 0 & 1 \\
b & a & 0
\end{array}\right)
$$

with $a, b \in k$.

Suppose $X_{H}$ is a $G$-regular semisimple element of $\mathfrak{h}(k)$ and $X \in$ $\mathfrak{g}^{r}(k)$ is an image of $X_{H}$, that is, $X_{H} \mapsto X$. It is a fact (see, for example, $[\mathbf{2 5}, \S 2])$ that the stable orbit of $X$ in $\mathfrak{g}(k)$ intersects $V(k)$ at exactly one point, say $X^{\prime}$. We let $\mu_{X^{\prime}}^{G}$ be the base point for the set of orbital integrals occurring in the right-hand side of the statement of the FL for the Lie algebra.

3.1.5. Ellipticity. There is one further reduction which may be made. An element $\gamma \in G^{r}(k)$ is called elliptic provided that $C_{G}(\gamma)(k)$ is compact. For example, if we suppose $p \neq 2$ and fix $\varepsilon \in R^{\times} \backslash\left(R^{\times}\right)^{2}$, then the element

$$
\left(\begin{array}{cc}
\varpi & 0 \\
0 & \varpi^{-1}
\end{array}\right)
$$

is not elliptic in $\mathrm{SL}_{2}(k)$, while the element

$$
\exp \left(\begin{array}{cc}
0 & \varpi \\
\varpi \varepsilon & 0
\end{array}\right)
$$

is. A $k$-torus is said to be elliptic provided that its group of $k$-points is compact.

As a warning to the reader, we comment that, unlike in the real or finite field situation, the number of $G(k)$-conjugacy classes of elliptic 
tori is, in general, enormous. For example, under suitable conditions on $k, \mathrm{SL}_{2}(k)$ has six, $\mathrm{Sp}_{34}(k)$ has

$$
3,329,926,392
$$

and $\operatorname{Sp}_{78}(k)$ has

$$
728,432,069,525,569,284
$$

$G(k)$-conjugacy classes of elliptic tori $[\mathbf{1}]$.

An endoscopic group $H$ is said to be elliptic provided that there exist $\gamma_{H} \in H^{r}(k)$ and elliptic $\gamma \in G^{r}(k)$ so that $\gamma_{H} \mapsto \gamma$. For example, the elliptic endoscopic group of $\mathrm{PGL}_{2}$ is $\mathrm{PGL}_{2}$. On the other hand, the elliptic endoscopic groups of $\mathrm{SL}_{2}$ are $\mathrm{SL}_{2}$ and $U_{E}^{1}$, while those of $\mathrm{G}_{2}$ are $\mathrm{G}_{2}, \mathrm{PGL}_{3}$, and $\mathrm{SO}_{4}$.

It follows from standard facts about parabolic induction that it is sufficient to verify the FL (on the Lie algebra) for the Lie algebras of elliptic endoscopic groups.

3.2. Asymptotic approach to the FL. In the early 1970s, Harish-Chandra and R. Howe studied the local behavior of the character of an irreducible smooth representation of $G(k)$. In particular, they established the Harish-Chandra-Howe local character expansion (introduced in Section 2.2.4). At the heart of their proofs was a remarkable finiteness statement, referred to as "Howe's conjecture", about $J(\mathfrak{g}(k))$, the space of $G(k)$-invariant distributions on $\mathfrak{g}(k)$.

It is natural to wonder how these finiteness results may be generalized and used in our context. In the 1990s, Waldspurger proved a very precise version, called a homogeneity result, of Howe's conjecture for "unramified classical groups". We need to introduce some notation before we can describe it.

3.2.1. Homogeneity results. One version of Howe's conjecture (which is a theorem) states that, given a compactly generated, closed, invariant subset $\omega$ of $\mathfrak{g}(k)$, there exists a lattice $L$ in $\mathfrak{g}(k)$ so that

$$
\operatorname{res}_{C_{c}(\mathfrak{g}(k) / L)} J(\omega)=\operatorname{res}_{C_{c}(\mathfrak{g}(k) / L)} J(\mathcal{N}) .
$$

Here, $J(\mathcal{N})$ denotes the span of the nilpotent orbital integrals (see $[\mathbf{3 7}]$ ), $J(\omega)$ denotes the subspace of distributions in $J(\mathfrak{g}(k))$ with support in $\omega$, and, if $T \in J(\mathfrak{g}(k))$, then $\operatorname{res}_{C_{c}(\mathfrak{g}(k) / L)} T$ denotes the restriction of $T$ to the space of functions in $C_{c}^{\infty}(\mathfrak{g}(k))$ which are invariant under translation by $L$.

The homogeneity result Waldspurger obtained can be described as follows. Let $\mathfrak{g}(k)_{0}$ denote the set of compact elements in $\mathfrak{g}(k)$. (For example, an element of $\mathfrak{s l}_{n}(k)$ is compact provided that each of its eigenvalues has nonnegative valuation.) We shall also need a Lie algebra version of the Iwahori-Hecke algebra. To a facet $F$ in the Bruhat-Tits building of $G(k)$, we can associate a parahoric subgroup $G(k)_{F}$ and its 
"Lie algebra" $\mathfrak{g}(k)_{F}$. For example, $\mathfrak{g}(R)=\mathfrak{g}(k)_{F}$ for some facet $F$. If $F$ is an (affine) chamber, then $G(k)_{F}$ is an Iwahori subgroup. Define

$$
\mathcal{H}:=\sum_{C} C_{c}\left(\mathfrak{g}(k) / \mathfrak{g}(k)_{C}\right)
$$

Here, the sum is over all chambers $C$ in the Bruhat-Tits building of $G$. We then have the following homogeneity result:

$$
\operatorname{res}_{\mathcal{H}} J\left(\mathfrak{g}(k)_{0}\right)=\operatorname{res}_{\mathcal{H}} J(\mathcal{N}) .
$$

Note that the characteristic function of $\mathfrak{g}(R)$ is an element of $\mathcal{H}$, and $\mu_{X}^{G}([\mathfrak{g}(R)])$ is nonzero only if $X \in \mathfrak{g}(k)_{0}$. Thus, from the homogeneity result, the FL can be viewed as a statement about the finite set of nilpotent orbital integrals. This divides the problem of verifying the FL into two subproblems: (1) Describe the stable distributions ${ }^{11}$ supported on the nilpotent cone of $\mathfrak{h}(k)$; and (2) For each such distribution, describe the corresponding ${ }^{12}$ distribution supported on the nilpotent cone of $\mathfrak{g}(k)$. Unfortunately, this approach has (at least) two problems (beyond the difficulty of doing (1) and (2)). First, in order to complete part (2), one needs to assume a version of the Matching Conjecture (see, for example, [24]). Second, for $X \in \mathfrak{g}^{r}(k) \cap \mathfrak{g}(k)_{0}$, it is known that

$$
\operatorname{res}_{\mathcal{H}} \mu_{X}^{G}=\sum_{\mathcal{O}} \Gamma_{\mathcal{O}}(X) \cdot \operatorname{res} \mathcal{H} \mu_{\mathcal{O}}
$$

where the sum runs over the nilpotent orbits in $\mathfrak{g}(k), \mu_{\mathcal{O}}$ is the nilpotent orbital integral corresponding to $\mathcal{O}$, and $\Gamma_{\mathcal{O}}$ is the Shalika germ attached to $\mathcal{O}$; but computing Shalika germs is not an easy task.

3.2.2. A brief description. Here is a brief description of how Waldspurger proceeds. He shows that, if $T \in J(\mathfrak{g}(k))$ is stable and $D$ is a linear combination of nilpotent orbital integrals satisfying

$$
\operatorname{res}_{\mathcal{H}} T=\operatorname{res}_{\mathcal{H}} D,
$$

then $D$ is stable. (Moreover, the sum of the summands in $D$ corresponding to orbits of a given fixed dimension is also a stable distribution.) This result is particularly useful because we have a very good understanding of where to find a basis for the dual of $\operatorname{res}_{\mathcal{H}} J\left(\mathfrak{g}(k)_{0}\right)$. Namely, fix an alcove $C$ in the Bruhat-Tits building of $G$. Define

$$
\mathcal{H}_{0}=\sum_{x \leq C} C\left(\mathfrak{g}(k)_{\{x\}} / \mathfrak{g}(k)_{C}\right) .
$$

${ }^{11}$ A distribution $T$ on $\mathfrak{g}(k)$ is said to be stable provided that, for $f \in C_{c}^{\infty}(\mathfrak{g}(k))$, we have $T(f)=0$ if $S \mu_{X}^{G}(f)=0$ for all $X \in \mathfrak{g}^{r}(k)$. Here $S \mu_{X}^{G}=\sum_{i} \mu_{X_{i}}^{G}$, where the sum is over representatives $X_{i}$ for the $G(k)$-conjugacy classes in the stable orbit of $X$. The measures are chosen to be compatible.

${ }^{12}$ Since we are no longer dealing with $G$-regular semisimple elements and their images in $\mathfrak{g}^{r}(k)$, we have to work to define the (conjectural) image (in the set of distributions on $\mathfrak{g}(k)$ ) of a stable distribution on the nilpotent cone of $\mathfrak{h}(k)$. 
Here the sum is over the vertices $x$ contained in the closure of $C$, and $C\left(\mathfrak{g}(k)_{\{x\}} / \mathfrak{g}(k)_{C}\right)$ denotes the subspace of $\mathcal{H}$ of functions with support in $\mathfrak{g}(k)_{\{x\}}$. Waldspurger proves that $\mathcal{H}_{0}$ contains a dual basis for $\operatorname{res}_{\mathcal{H}} J\left(\mathfrak{g}(k)_{0}\right)$ - by looking at reductive quotients, this result naturally leads to questions about finite groups of Lie type.

Because Lusztig's generalized Green functions (see, for example, [32]) separate nilpotent orbits in the Lie algebra of a finite group of Lie type, they naturally arise in the present context. Indeed, a basis for the dual of $\operatorname{res}_{\mathcal{H}} J\left(\mathfrak{g}(k)_{0}\right)$ may be obtained by taking the Fourier transform of the inflation of each generalized Green function which occurs on the Lie algebra of the reductive quotient of a parahoric. Waldspurger associates to each such generalized Green function $\mathcal{G}$ a combination $T_{\mathcal{G}}$ of regular semisimple orbital integrals on $\mathfrak{g}(k)$. This is good because the definition of stability for distributions is phrased in terms of regular semisimple orbital integrals. He is then able to form stable combinations of the $T_{\mathcal{G}}$, and thus obtains the stable distributions in $J(\mathcal{N})$.

To calculate the image in $J(\mathcal{N})$ of a stable distribution in $J\left(\mathcal{N}_{\mathfrak{h}}\right)$, the set of nilpotent elements in the Lie algebra of an (elliptic) endoscopic group $H$, Waldspurger first explicitly calculates the transfer factors. From the previous paragraph, we have explicit combinations of stable (regular semisimple) orbital integrals on $\mathfrak{h}(k)$ which have nice properties with respect to a basis for the stable distributions supported on $\mathcal{N}_{\mathfrak{h}}$. Pick one such basis element, say $T$, and consider the associated combination of stable orbital integrals on $\mathfrak{h}(k)$. Waldspurger explicitly calculates the (conjectural) image of this combination of stable orbital integrals, and then relates this image to a combination of more familiar objects with which it agrees when restricted to $\mathcal{H}$. He then invokes the above homogeneity result to identify the (conjectural) image of $T$ in $J(\mathcal{N})$.

3.3. Geometric results. We discuss some results obtained in the positive-characteristic setting using geometry. It has recently been announced that these techniques have been used to prove the FL [35]. I believe that most of these approaches have their origins in the work of Kazhdan and Lusztig [20]. Since Waldspurger [46] has shown that the validity of the FL in positive characteristic implies its validity in characteristic zero (and vice versa), this approach is relevant for the FL in our setting.

Drop all previous notation.

Let $\mathfrak{f}$ denote a finite field, and suppose $G$ is a connected reductive $\mathfrak{f}$-group with Lie algebra $\mathfrak{g}$. Choose a prime $\ell$ different from the characteristic of $\mathfrak{f}$. Let $X$ denote a smooth projective curve defined over $\mathfrak{f}$ and $F=\mathfrak{f}(X)$ the field of rational functions on $X$. For each place $\nu$ of $F$, let $\mathcal{O}_{\nu}$ denote the completion of $\mathcal{O}_{X, \nu}$ and $F_{\nu}$ the corresponding completion of $F$. Let $\mathbb{A}_{F}$ denote the adèle ring of $F$ and $G\left(\mathbb{A}_{F}\right)$ the 
corresponding group of adèlic points of $G$. We set $G(\hat{\mathcal{O}}):=\prod_{\nu} G\left(\mathcal{O}_{\nu}\right)$. We fix an effective divisor $D=\sum_{\nu} d_{\nu} \nu$ (of large degree).

The idea of the geometric approach is to use the Grothendieck dictionary relating $\overline{\mathbb{Q}}_{\ell}$-valued functions on $X$ to sheaves on $X$ by taking the trace of Frobenius on stalks. Thus, we look for a proof of the FL which finds an equality between two (perverse) sheaves for which the corresponding functions are the two sides of the FL. Initially, this approach to the problem produced a solution in two cases: by Kottwitz, Goresky, and MacPherson in the equi-valued case $[\mathbf{8}, \mathbf{9}]$, and by Laumon and Ngô for unitary groups [31]. The main obstruction to this approach concerns the "purity" of the objects under consideration. For Laumon and Ngô, this difficulty was avoided by first proving a global result, and then deducing the local result from it.

Our goal in what follows is to give some fairly vague idea of how the approach of Ngô and Laumon proceeds. To do this, we need to understand the object

$$
\mathcal{M}=\left\{(E, \varphi) \mid E \rightarrow X \text { is a G-torsor, } \varphi \in \mathrm{H}^{0}\left(X, \operatorname{ad}(E) \otimes \mathcal{O}_{X}(D)\right)\right\},
$$

and "count" the number of rational elements in $\mathcal{M}$. (Terminology is defined below.)

3.3.1. G-torsors. In this section, we allow $\mathfrak{f}$ to be any field.

A $G$-torsor, to be denoted $E \rightarrow X$, is a smooth $\mathfrak{f}$-variety $E$ endowed with an action $G \times E \rightarrow E$ and an $\mathfrak{f}$-morphism $E \rightarrow X$ so that

(1) the map $E \rightarrow X$ is flat and surjective, and

(2) the action map $G \times E \rightarrow E \times_{X} E$ is an isomorphism.

The second condition may be thought of as saying that $G$ acts simply transitively on each fiber of the map $E \rightarrow X$.

EXAMPLE 3.3.1. Take $E=G \times X$. Then projection on the second factor gives us the $G$-torsor $G \times X \rightarrow X$.

Two $G$-torsors $E \rightarrow X$ and $E^{\prime} \rightarrow X$ are isomorphic provided that there exists a $G$-equivariant $X$-morphism $\varphi: E \rightarrow E^{\prime}$.

A $G$-torsor $E \rightarrow X$ is said to be trivial provided that it is isomorphic to the $G$-torsor $G \times X \rightarrow X$ of Example 3.3.1. Equivalently, it is trivial if there is a section $s: X \rightarrow E$.

From a slight modification of the material in [40, III, $\S 1.3]$, the set of isomorphism classes of $G$-torsors of the form $E \rightarrow \operatorname{spec}(\mathfrak{f})$ is isomorphic to $\mathrm{H}^{1}(\mathfrak{f}, G)$.

3.3.2. Application to our setting. We again assume that $\mathfrak{f}$ is a finite field.

Suppose $E \rightarrow X$ is a $G$-torsor. For a place $\nu$ of $F$, we consider the $G$-torsor $E \otimes \mathcal{O}_{X, \nu} \rightarrow \operatorname{spec}\left(\mathcal{O}_{X, \nu}\right)$. Using Lang's "trick" [27] (see also, $[4, \S 16]$ ), we see that the special fiber $E_{\nu} \rightarrow\{\nu\}$ is a trivial $G$ torsor. Since $E \rightarrow X$ is a smooth morphism and $\mathcal{O}_{\nu}$ is Henselian, it 
follows that the $G$-torsor $E \otimes \mathcal{O}_{\nu} \rightarrow \operatorname{spec}\left(\mathcal{O}_{\nu}\right)$ admits a section. Thus, the $G$-torsor $E_{F_{\nu}} \rightarrow \operatorname{spec}\left(F_{\nu}\right)$ is trivial.

Consider the natural map

$$
\mathrm{H}^{1}(F, G) \rightarrow \prod_{\nu} \mathrm{H}^{1}\left(F_{\nu}, G\right) .
$$

From the discussion above, the generic fiber $E_{F} \rightarrow \operatorname{spec}(F)$ associated to a $G$-torsor $E \rightarrow X$ lies in the group $\operatorname{ker}^{1}(F, G):=\operatorname{ker}\left(\mathrm{H}^{1}(F, G) \rightarrow\right.$ $\left.\prod_{\nu} \mathrm{H}^{1}\left(F_{\nu}, G\right)\right)$.

If $E \rightarrow X$ is a $G$-torsor with trivial generic fiber (i.e., $E_{F} \rightarrow \operatorname{spec}(F)$ is trivial), then, from the above discussion, there is an element $s \in$ $E_{F}(F)$, and, for each place $\nu$, an element $e_{\nu} \in E\left(\mathcal{O}_{\nu}\right) \subset E\left(F_{\nu}\right)$. Since $E(F) \subset E\left(F_{\nu}\right)$ for all $\nu$, we can try to compare $s$ and $e_{\nu}$ at all places. That is, we can try to measure how far $E \rightarrow X$ is from being trivial. For every $\nu$ there is a unique $g_{\nu} \in G\left(F_{\nu}\right)$ such that $s=g_{\nu} e_{\nu}$ in $E\left(F_{\nu}\right)$; and, for almost all $\nu$, we have $g_{\nu} \in G\left(\mathcal{O}_{\nu}\right)$ (since $s, e_{\nu} \in E\left(\mathcal{O}_{\nu}\right)$ for almost all $\nu)$. Thus, $\left(g_{\nu}\right)_{\nu} \in G\left(\mathbb{A}_{F}\right)$. Its projection into $G(F) \backslash G\left(\mathbb{A}_{F}\right) / G(\hat{\mathcal{O}})$ is independent of all choices. Moreover, the map $(E \rightarrow X) \mapsto\left(g_{\nu}\right)_{\nu}$ induces a bijective correspondence between the set of (isomorphism classes of) $G$-torsors over $X$ with trivial generic fiber and the double coset space

$$
G(F) \backslash G\left(\mathbb{A}_{F}\right) / G(\hat{\mathcal{O}}) .
$$

3.3.3. Completing the definition of $\mathcal{M}$. From the $G$-torsor $E \rightarrow X$ we may construct the $\operatorname{GL}(\mathfrak{g})$-torsor $\operatorname{Ad}(E) \rightarrow X$ by setting $\operatorname{Ad}(E):=$ $\left(E \times \times_{X} \mathrm{GL}(\mathfrak{g})\right) / G$. (Here $G$ acts so that, in the quotient, " $(g e, h)=$ $\left(e, \operatorname{Ad}\left(g^{-1}\right) h\right)$ ".) By the equivalence between $\mathrm{GL}_{n}$-torsors and vector bundles, the $\mathrm{GL}(\mathfrak{g})$-torsor $\operatorname{Ad}(E) \rightarrow X$ is trivial Zariski-locally over $X$ (and not merely étale-locally on $X$, as is the case for torsors under most smooth algebraic groups). We denote by $\operatorname{ad}(E)$ the Zariski $\mathfrak{g}$-bundle over $X$ which corresponds to the $\mathrm{GL}(\mathfrak{g})$-torsor $\operatorname{Ad}(E) \rightarrow X$.

Recall that $\mathcal{O}_{X}(D)$ is the sheaf which associates to a Zariski-open subset $U$ of $X$ the set of $f \in F=\mathfrak{f}(X)$ for which $\operatorname{ord}_{\nu}(f) \geq-d_{\nu}$ for all $\nu \in U$. We then have that

$$
\mathrm{H}^{0}\left(X, \operatorname{ad}(E) \otimes \mathcal{O}_{X}(D)\right)
$$

is the (finite-dimensional) vector space of sections of $\operatorname{ad}(E) \otimes \mathcal{O}_{X}(D) \rightarrow$ $X$. A calculation with the definitions show that $\mathrm{H}^{0}\left(X, \operatorname{ad}(E) \otimes \mathcal{O}_{X}(D)\right)$ may be characterized as

$$
\left\{Y \in \mathfrak{g}(F): \operatorname{Ad} g_{\nu}^{-1} Y \in \varpi_{\nu}^{-d_{\nu}} \mathfrak{g}\left(\mathcal{O}_{\nu}\right) \text { for all } \nu\right\}
$$

(recall that we have associated $\left(g_{\nu}\right)_{\nu} \in G\left(\mathbb{A}_{F}\right)$ to $E$ ).

3.3.4. The end. We let $J_{D}$ denote the principal ideal in $\mathbb{A}_{F}$ generated by an idèle having the local orders prescribed by $D$. We let $\left[L_{D}\right]$ denote the characteristic function of the subset $L_{D}=J_{D}\left(\hat{\mathcal{O}} \otimes_{\mathfrak{f}} \mathfrak{g}\right)$ of $\mathbb{A}_{F} \otimes_{\mathfrak{f}} \mathfrak{g}$. 
Recall that we wish to "compute" the number of elements in $\mathcal{M}(\mathfrak{f})$. This set does not, in general, have finite cardinality. However, ignoring this, one can make formal calculations which rather convincingly suggest this is a reasonable direction to look for a proof of the FL on the Lie algebra. All of the equalities below should be taken with a grain of salt. Also, at the end of the heuristic, I have swept all of the very detailed analysis which goes into stabilizing the elliptic part of the trace formula $[\mathbf{2 3}]$ under the rug.

In each integral below, the measure $d g^{*}$ is the appropriate quotient measure. From our discussions above, one obtains

$$
|\mathcal{M}(\mathfrak{f})|=\left|\operatorname{ker}^{1}(F, G)\right| \sum_{Y \in \mathfrak{g}(F)} \int_{G(F) \backslash G\left(\mathbb{A}_{F}\right)}\left[L_{D}\right]\left(\operatorname{Ad}\left(g^{-1}\right) Y\right) d g^{*}
$$

Recalling that we are ignoring convergence issues, one may formally rewrite the above as

$$
\begin{aligned}
& |\mathcal{M}(\mathfrak{f})| \\
& =\left|\operatorname{ker}^{1}(F, G)\right| \sum_{Y \in \mathfrak{g}(F) / G(F)} \sum_{h \in C_{G}(Y)(F) \backslash G(F)} \int_{G(F) \backslash G\left(\mathbb{A}_{F}\right)}\left[L_{D}\right]\left(\operatorname{Ad}\left(g^{-1} h^{-1}\right) Y\right) d g^{*} \\
& =\left|\operatorname{ker}^{1}(F, G)\right| \sum_{Y \in \mathfrak{g}(F) / G(F)} \int_{C_{G}(Y)(F) \backslash G\left(\mathbb{A}_{F}\right)}\left[L_{D}\right]\left(\operatorname{Ad}\left(g^{-1}\right) Y\right) d g^{*} \\
& =\left|\operatorname{ker}^{1}(F, G)\right| \sum_{Y \in \mathfrak{g}(F) / G(F)} \operatorname{vol}\left(C_{G}(Y)(F) \backslash C_{G}(Y)\left(\mathbb{A}_{F}\right)\right) \mu_{Y}\left(\left[L_{D}\right]\right),
\end{aligned}
$$

where

$$
\mu_{Y}\left(\left[L_{D}\right]\right)=\int_{C_{G}(Y)\left(\mathbb{A}_{F}\right) \backslash G\left(\mathbb{A}_{F}\right)}\left[L_{D}\right]\left(\operatorname{Ad}\left(g^{-1}\right) Y\right) d g^{*} .
$$

This is reminiscent of the geometric side of the invariant trace formula. If we could somehow modify $\mathcal{M}$ so that the sum above ran over only elliptic regular semisimple $Y$ (that is, those $Y \in G(F)$ for which $C_{G}(Y)$ is an $F$-anisotropic torus), then we'd be looking at the Lie algebra version of the regular elliptic part of the invariant trace formula (see, for example, $[\mathbf{2}, \S 27]$ or $[\mathbf{2 3}]$ ). So, after some tweaking to isolate the regular elliptic part, one hopes that $|\mathcal{M}(\mathfrak{f})|$ would be given by a formula like

$$
\text { const } \sum_{Y \in \mathfrak{g}_{\mathrm{ell}}(F) / G(F)} \sum_{\left.\kappa \in \pi_{0}\left(\widehat{C_{G}(Y)}\right)^{\Gamma}\right)} \mu_{Y}^{\kappa}\left(\left[L_{D}\right]\right)
$$

where const is a constant, $\mathfrak{g}_{\text {ell }}(F)$ is the set of regular elliptic elements in $\mathfrak{g}(F)$, and

$$
\mu_{Y}^{\kappa}\left(\left[L_{D}\right]\right)=\prod_{\nu} \sum_{Y^{\prime} \sim Y} \kappa\left(Y^{\prime}\right) \cdot \mu_{Y^{\prime}}^{G}\left(\left[\varpi_{\nu}^{d_{\nu}} \mathfrak{g}\left(\mathcal{O}_{\nu}\right)\right]\right)
$$

is a product of $\kappa$-orbital integrals (see Section 2.4.2). So, counting the points on $\mathcal{M}(\mathfrak{f})$ has brought us back to the FL. 


\section{References}

[1] J. Adler, personal communication to Paul J. Sally, Jr..

[2] J. Arthur, An introduction to the trace formula, Harmonic analysis, the trace formula, and Shimura varieties, 1-263, Clay Math. Proc., 4, Amer. Math. Soc., Providence, RI, 2005.

[3] A. Borel, Automorphic L-functions, Automorphic forms, representations and $L$-functions (Proc. Sympos. Pure Math., Oregon State Univ., Corvallis, Ore., 1977), Part 2, 27-61, Proc. Sympos. Pure Math., XXXIII, Amer. Math. Soc., Providence, RI, 1979.

[4] A. Borel, Linear algebraic groups, Second edition. Graduate Texts in Mathematics, 126, Springer-Verlag, New York, 1991.

[5] R. Carter, Finite groups of Lie type. Conjugacy classes and complex characters, Reprint of the 1985 original, John Wiley \& Sons, Ltd., Chichester, 1993.

[6] W. Casselman, Introduction to the theory of admissible representations of p-adic reductive groups, 1995.

[7] S. DeBacker and M. Reeder, Depth-zero supercuspidal L-packets and their stability, Ann. of Math., to appear.

[8] M. Goresky, R.E. Kottwitz, and R. MacPherson, Homology of affine Springer fibers in the unramified case, Duke Math. J. 121(3) (2004) 509-561.

[9] M. Goresky, R.E. Kottwitz, and R. MacPherson, Purity of equivalued affine Springer fibers, Represent. Theory 10 (2006) 130-146.

[10] D. Gross and M. Reeder, From Laplace to Langlands via representations of orthogonal groups, Bull. Amer. Math. Soc. (N.S.) 43(2) (2006) 163-205.

[11] T. Hales, A simple definition of transfer factors for unramified groups, Representation theory of groups and algebras, 109-134, Contemp. Math., 145, Amer. Math. Soc., Providence, RI, 1993.

[12] T. Hales, On the fundamental lemma for standard endoscopy: reduction to unit elements, Canad. J. Math. 47(5) (1995) 974-994.

[13] T. Hales, A statement of the fundamental lemma, Harmonic analysis, the trace formula, and Shimura varieties, 643-658, Clay Math. Proc., 4, Amer. Math. Soc., Providence, RI, 2005.

[14] Harish-Chandra, The characters of reductive p-adic groups, Contributions to algebra, 175-182, Academic Press, New York, 1977.

[15] Harish-Chandra, Admissible invariant distributions on reductive p-adic groups, preface and notes by Stephen DeBacker and Paul J. Sally, Jr., University Lecture Series, 16, Amer. Math. Soc., Providence, RI, 1999.

[16] J. Humphreys, Conjugacy classes in semisimple algebraic groups, Mathematical Surveys and Monographs, 43, Amer. Math. Soc., Providence, RI, 1995.

[17] H. Jacquet, Sur les représentations des groupes réductifs p-adiques, C. R. Acad. Sci. Paris Sr. A-B 280 (1975), Aii, A1271-A1272.

[18] H. Jacquet and R. P. Langlands, Automorphic forms on GL(2), Lecture Notes in Mathematics, 114, Springer-Verlag, Berlin-New York, 1970.

[19] D. Kazhdan, On lifting, Lie group representations, II, 209-249, Lecture Notes in Math., 1041, Springer, Berlin, 1984.

[20] D. Kazhdan and G. Lusztig, Fixed point varieties on affine flag manifolds, Israel J. Math. 62(2) (1988) 129-168.

[21] R.E. Kottwitz, Rational conjugacy classes in reductive groups, Duke Math. J. 49(4) (1982) 785-806.

[22] R.E. Kottwitz, Stable trace formula: cuspidal tempered terms, Duke Math. J. 51(3) (1984) 611-650.

[23] R.E. Kottwitz, Stable trace formula: elliptic singular terms, Math. Ann. 275(3) (1986) 365-399. 
[24] R.E. Kottwitz, Harmonic analysis on semisimple p-adic Lie algebras, Proceedings of the International Congress of Mathematicians, Vol. II (Berlin, 1998), Doc. Math. 1998, Extra Vol. II, 553-562

[25] R.E. Kottwitz, Transfer factors for Lie algebras, Represent. Theory 3 (1999) $127-138$.

[26] R.E. Kottwitz and D. Shelstad, Foundations of twisted endoscopy, Astérisque 255 (1999).

[27] S. Lang, Algebraic groups over finite fields, Amer. J. Math. 78 (1956) 555-563.

[28] R.P. Langlands, Les débuts d'une formule des traces stable, Publications Mathématiques de l'Université Paris VII, 13, Université de Paris VII, U.E.R. de Mathématiques, Paris, 1983.

[29] R.P. Langlands and D. Shelstad, On the definition of transfer factors, Math. Ann. 87 (1987) 219-271.

[30] R.P. Langlands and D. Shelstad, Descent for transfer factors, The Grothendieck Festschrift, Vol. II, 485-563, Progr. Math., 87, Birkhuser Boston, Boston, MA, 1990.

[31] G. Laumon and B.C. Ngô, Le lemme fondamental pour les groupes unitaires, preprint, 2004.

[32] G. Lusztig, Green functions and character sheaves, Ann. of Math. (2) 131(2) (1990) 355-408.

[33] C. Møglin and J.-L. Waldspurger, Modèles de Whittaker dégénérés pour des groupes p-adiques, Math. Z. 196(3) (1987) 427-452.

[34] A. Moy and G. Prasad, Unrefined minimal K-types for p-adic groups, Invent. Math. 116(1-3) (1994) 393-408.

[35] B.C. Ngô, Le Lemme Fondamental pour les Algèbres de Lie, preprint, March, 2007.

[36] M.S. Raghunathan, Tori in quasi-split-groups, J. Ramanujan Math. Soc. 19(4) (2004) 281-287.

[37] R. Ranga Rao, Orbital integrals in reductive groups, Ann. of Math. (2) 96 (1972) 505-510.

[38] F. Rodier, Modèle de Whittaker et caractères de représentations, Non-commutative harmonic analysis (Actes Colloq., Marseille-Luminy, 1974), 151-171, Lecture Notes in Math., Vol. 466, Springer, Berlin, 1975.

[39] F. Rodier, Sur le caractère de Steinberg, Compositio Math. 59(2) (1986) 147149.

[40] J.-P. Serre, Galois cohomology, Springer Monographs in Mathematics, SpringerVerlag, Berlin, 2002.

[41] F. Shahidi, A proof of Langlands' conjecture on Plancherel measures; complementary series for p-adic groups, Ann. of Math. (2) 132(2) (1990) 273-330.

[42] D. Shelstad, A formula for regular unipotent germs, Orbites unipotentes et représentations, II, Astérisque 171-172 (1989) 275-277.

[43] L. Spice, Topological Jordan decompositions, 2006, preprint.

[44] J.-L. Waldspurger, Une formule des traces locale pour les algèbres de Lie padiques, J. Reine Angew. Math. 465 (1995) 41-99.

[45] J.-L. Waldspurger, Le lemme fondamental implique le transfert, Compositio Math. 105(2) (1997) 153-236.

[46] J.-L. Waldspurger, Endoscopie et changement de caractéristique, J. Inst. Math. Jussieu 5(3) (2006) 423-525.

Department of Mathematics, University of Michigan, 2074 East Hall, 530 Church St., Ann Arbor, MI 48109-1043

E-mail address: smdbackr@umich.edu 
